\title{
Major Adverse Renal and Cardiovascular Events following Intra-Arterial Contrast Media Administration in Hospitalized Patients with Comorbid Conditions
}

\author{
Peter McCullough ${ }^{a}$ Chaan S. Ng ${ }^{b}$ Michael Ryan ${ }^{c}$ Erin R. Baker ${ }^{d}$ \\ Ravindra Mehta ${ }^{\mathrm{e}}$

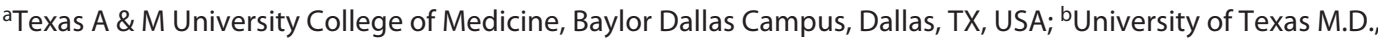 \\ Anderson Cancer Center, Houston, TX, USA; ${ }^{C}$ MPR Consulting, Cincinnati, OH, USA; ${ }^{\mathrm{d}} \mathrm{CTI}$ Clinical Trial and Consulting

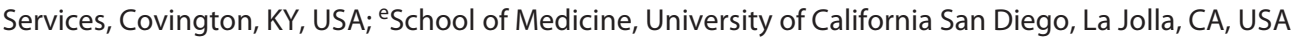

\section{Keywords \\ Contrast media - Major adverse cardiovascular events . \\ Major adverse renal events . Chronic kidney disease . \\ Chronic total occlusion}

\begin{abstract}
Introduction: Several clinical studies and meta-analyses have demonstrated lower incidence of adverse renal and cardiovascular outcomes associated with the use of iso-osmolar contrast media (IOCM) than low-osmolar contrast media (LOCM) in patients with variable risk profiles undergoing intra-arterial interventional procedures. However, the association of contrast-type and major adverse renal and cardiovascular events (MARCE) has not been studied via comprehensive and robust real-world data analyses in patients with comorbid conditions considered at risk for post-procedural acute kidney injury (AKI). The objective of this study was therefore to retrospectively assess the MARCE rates comparing IOCM with LOCM in at-risk patients receiving iodinated intra-arterial contrast media using a real-world inpatient data source. Methods: Patients who underwent a diagnostic or treatment procedure with intra-arterial IOCM or LOCM administration were identified using the Premier Healthcare
\end{abstract}

karger@karger.com www.karger.com/crm

Karger ${ }^{\prime \prime} \div$
(C) 2021 The Author(s)

Published by S. Karger AG, Basel

This is an Open Access article licensed under the Creative Common Attribution-NonCommercial-4.0 International License (CC BY-NC) (http://www.karger.com/Services/OpenAccessLicense), applicable to the online version of the article only. Usage and distribution for commercial purposes requires written permission.
Database. Patient subgroups including those with diabetes, heart failure, chronic kidney disease (CKD) stages 1-4, CKD $3-4$, or diagnosis of chronic total occlusion (CTO) were formed. Subgroups with combinations of diabetes and CKD 3-4 with and without CTO were also investigated. We compared the primary endpoint of MARCE (composite of AKI, AKI requiring dialysis, acute myocardial infarction, stroke/transient ischemic attack, stent occlusion/thrombosis, or death) after IOCM versus LOCM administration via adjusted multivariable regression analyses. Results: A total of 536,013 inpatient visits met the primary inclusion and exclusion criteria (IOCM $=133,192 ;$ LOCM $=402,821)$. After multivariable modeling, the use of IOCM was associated with a significantly lower incidence of MARCE than LOCM in patients with CKD $1-4$, CKD 3-4, diabetes, or heart failure, with greatest absolute risk reduction (ARR) of 2.4\% $(p<0.0001)$ in CKD 3-4 patients (relative risk reduction $[R R R]=13.8 \%$, number needed to treat $[N N T]=43$ ). Additionally, ARR associated with IOCM increased to $3.5 \%(p<0.0001)$ in patients with combined comorbidities of diabetes and CKD 3-4 (RRR $=19.1 \%$, NNT = 29). Statistically significant risk reduction was also found for the use of IOCM among patients who underwent revascularization for CTO (ARR $=1.6 \%[p<0.0001]$, RRR $=22.3 \%$, NNT $=62)$. Conclusion: Intra-arterial administration using IOCM 
in at-risk patients is associated with lower rates of MARCE in patients with a combination of CKD 3-4 and diabetes and with meaningful NNT in favor of IOCM.

(C) 2021 The Author(s).

Published by S. Karger AG, Basel than the use of LOCM. This difference is especially apparent in patients with CTO, providing real-world data validation

in patients with comorbid conditions including one or more risk factors for post-procedural AKI undergoing intra-arterial contrast-enhanced procedures. Therefore, the objective of this study was to retrospectively assess the rate of MARCE comparing IOCM with LOCM in patients at risk for AKI receiving iodinated contrast media intra-arterially using a real-world inpatient data source.

\section{Methods}

Acute kidney injury (AKI) is an abrupt decrease in kidney function which is often caused by critical events such as sepsis, critical illness, circulatory shock, trauma, or major surgery but can also be triggered by exposure to additional risk factors such as nephrotoxic medications or iodinated contrast media $[1,2]$. When AKI develops shortly following contrast media use, it is termed postcontrast (PC-), contrast-associated (CA-) or contrast-induced (CI-) AKI, the latter if considered causally related. For patients undergoing diagnostic or interventional procedures requiring intra-arterial administration of iodinated contrast media, AKI is a potential adverse event [2] associated with downstream outcomes such as bleeding, myocardial infarction, and death $[3,4]$.

The incidence of AKI following intra-arterial contrast administration varies, and can be $>50 \%$ in high-risk patient groups typically comprising patients with pre-existing renal dysfunction and concurrent diabetes [5]. Higher AKI rates are also correlated with the administration of larger volumes of contrast media [6] that are often needed in complex high-risk interventional procedures, including multi-vessel percutaneous coronary interventions and revascularization procedures in patients with chronic total occlusion (CTO) [7].

Iso-osmolar contrast media (IOCM) has been associated with reduced adverse renal and cardiovascular outcomes compared with low-osmolar contrast media (LOCM) in prior randomized controlled trials $[8,9]$ and meta-analyses [10-12], and particularly in at-risk patients with pre-existing renal dysfunction $[8,9]$ and diabetes [12]. In addition to randomized controlled trials and meta-analyses, a recent analysis of real-world data [13] from the Premier Healthcare Database performed on 333,533 inpatient "all-comers" undergoing coronary, peripheral or carotid angioplasty showed significantly lower rates of major adverse renal and cardiovascular events (MARCE) with IOCM than multiple LOCM pooled together, with 9.32\% lower relative risk of MARCE associated with the use of IOCM. However, this association has not been studied via large-scale real-world data analyses
Data from the Premier Healthcare Database spanning July 2012-December 2018 were used for this analysis. The database graphic characteristics, comorbidities, and interventional procedures representing $\sim 25 \%$ of all inpatient admissions in the USA [14]. From this set, inpatient visits with a procedure that required intra-arterial administration of either low-osmolar or iso-osmolar contrast media were included in the analysis (online suppl. Table A; for all online suppl. material, see www.karger.com/ doi/10.1159/000517884). Patients who had chronic kidney disease (CKD) stage 5 or end-stage renal disease were excluded (Fig. 1).

\section{Study Inclusion Criteria}

Inpatient hospital visits which included a diagnostic or treatment procedure with intra-arterial administration of iodinated contrast media were included. If the visit also included intravenous delivery of contrast, it was excluded. Procedures of interest included coronary and non-coronary stent placement, angioplasty, atherectomy, heart valve and related procedures, other operations on the heart and pericardium or vessels, embolization, or removal of intraluminal device (see online suppl. Table A for complete code listing).

Subgroups based on comorbid conditions including diabetes, heart failure, CKD 1-4, CKD 3-4, or diagnosis of CTO were formed (see online suppl. Table B for complete code listing). Groups with combinations of diabetes and CKD 3-4 with and without CTO were also investigated. Diabetes and heart failure subgroups were developed from the Elixhauser Comorbidity Index (ECI) score comorbidity categories [15]. The ECI score includes 31 categories of comorbidities associated with mortality. These comorbidities were identified using diagnosis codes from the admission for the inpatient procedure of interest. A composite ECI score was calculated from the comorbidity categories (see online suppl. Table $\mathrm{C}$ for complete code listing). The CTO and CKD groups were formed based on ICD-9 and ICD-10 diagnosis codes (see online suppl. Table B).

\section{Predictors and Outcome Variables}

The primary outcome of interest was the composite end point of MARCE, inclusive of the following (1) renal events - renal failure with dialysis or acute kidney injury (including CI-AKI) with or without dialysis, (2) acute myocardial infarction (AMI), (3) stroke/transient ischemic attack, (4) stent occlusion/thrombosis, or (5) death (see online suppl. Table D for coding detail). In order to qualify as an outcome during the hospital admission, any adverse event was required to be absent on admission and present at discharge. contains administrative claims data, which include sociodemo- 
Fig. 1. Attrition diagram of inpatient records. CKD, chronic kidney disease; ESRD, end-stage renal disease; IOCM, iso-osmolar contrast media; LOCM, low-osmolar contrast media.

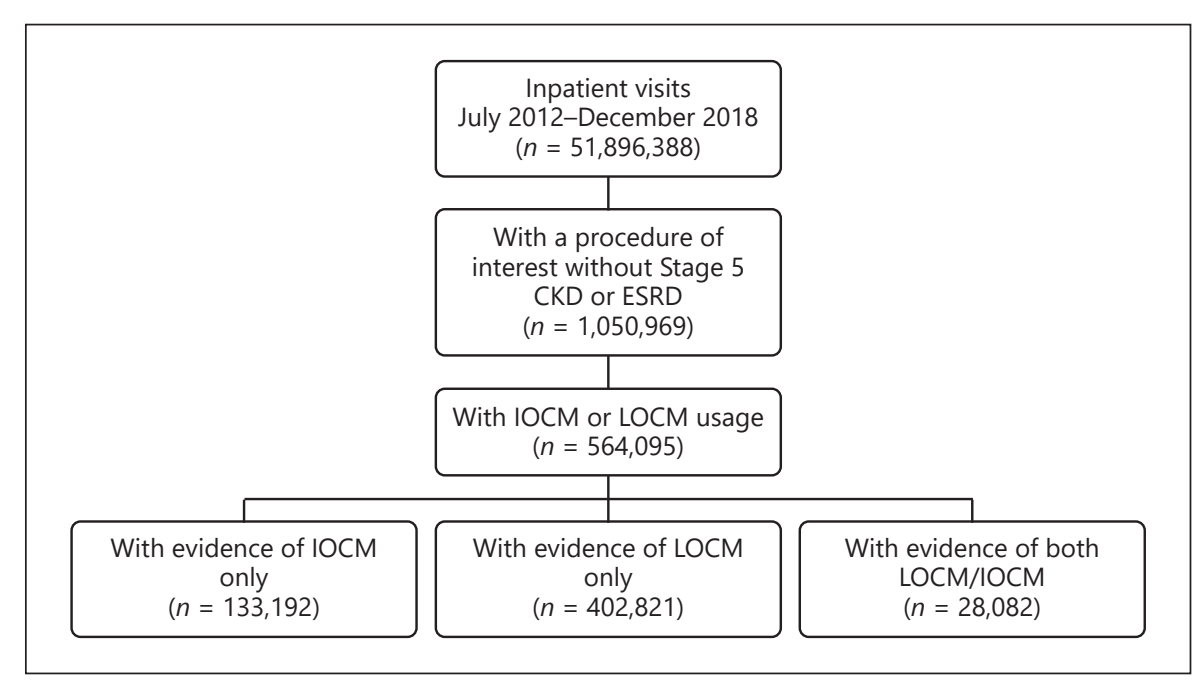

Using the Premier Standard Charge Master (which is a comprehensive table of items billable to a patient or health insurance provider), we identified IOCM (iodixanol) and LOCM (iohexol, ioversol, iopamidol, and other) contrast media. IOCM (vs. LOCM) was the main exposure or independent variable of interest.

Other independent variables of interest included patient demographics, comorbid conditions, and admission status. Patient demographics for this analysis included age, race, sex and year. Comorbid conditions were measured with a composite ECI score calculated from the comorbidity categories [15]. Hospital admission status was also considered. In the database admission status is categorized as follows: emergency (patient requires immediate medical intervention and is typically admitted through the emergency room), urgent (patient requires immediate attention and is generally admitted to the first available/suitable accommodation), trauma center (visit is to a designated trauma center), other or unknown (status is not known) and elective (patient's condition permits adequate time to schedule suitable accommodations).

\section{Statistical Analysis}

Multivariable regression analysis was conducted using the hospital fixed-effects specification to assess the relationship between MARCE events and type of contrast agent used. The hospital fixedeffects specification method was used to control for observable and unobservable differences in patients (severity of comorbid conditions and other hospital factors such as surgical practices, treatments, staffing patterns, physician skill, etc.) across hospitals that may influence choice of contrast medium and associated outcomes. The multivariable regression model was run for each comorbidity-based group and adjusted for year, patient demographics (age, sex, admission status, and race), and ECI. The model also adjusted for CKD status for all comorbidity groups except for the CKD subgroup itself. The absolute risk reduction (ARR) is the reduction in absolute risk of MARCE when IOCM rather than LOCM is used and was calculated via the multivariable modeling described above. The relative risk reduction (RRR) is calculated by dividing the ARR by the incidence of MARCE in the corresponding LOCM cohort. The number needed to treat (NNT) is the inverse of the ARR rounded up to the nearest whole number and represents the number of patients required to receive IOCM instead of LOCM to avoid each additional MARCE event. All statistical analyses in this study were performed using SAS software, version 9.4 (SAS Institute, Inc., Cary, NC, USA).

\section{Results}

Among the identified 1,050,969 inpatient visits with a procedure of interest and without CKD stage 5 or ESRD, those with a record of IOCM $(12.7 \%, N=133,192)$ or LOCM $(38.3 \%, N=402,821)$ usage were included in the final analyzable dataset. Patient visits with evidence of both IOCM and LOCM $(2.7 \%, N=28,082)$ were excluded as well as visits without recorded use of either type of contrast medium (Fig. 1).

Among these inpatient visits, subgroups including patients with 1 or more specific risk factors were identified. Within the overall patient cohort, there were 202,189 (37.7\%) patients with diabetes, 116,764 (21.8\%) with heart failure, and 83,243 (15.5\%) patients with CKD of which 48,639 (9.1\%) patients were in CKD stages 3-4. There were 78,254 (14.6\%) diagnoses of CTO. Demographics, ECI score, and percentage with CKD status are reported in Table 1. Additional descriptive variables including insurance type, admission type, and year of the procedure of interest are reported in online suppl. Table E, with breakdown of comorbidities in online suppl. Table F. More patients were administered LOCM than IOCM in the individual risk cohorts; however, IOCM use was higher than LOCM use in the combined risk groups comprising CKD 3-4 and diabetes (29,951 patients) and CKD 3-4, diabetes and CTO diagnosis (5,824 patients). 
Table 1. Demographics for comorbidity groups of interest in procedures involving intra-arterial contrast medium administration

\begin{tabular}{|c|c|c|c|c|c|c|c|c|c|c|}
\hline Cohort & \multicolumn{5}{|l|}{ LOCM } & \multicolumn{5}{|l|}{ IOCM } \\
\hline Diabetes & 148,170 & $65.5 \pm 11.7$ & $94,198(63.6)$ & $4.3 \pm 2.0$ & 14.8 & 54,019 & $68.0 \pm 11.6$ & $34,193(63.3)$ & $4.8 \pm 2.0$ & 26.2 \\
\hline Heart failure & 83,740 & $69.5 \pm 12.5$ & $52,375(62.5)$ & $5.3 \pm 2.0$ & 21.6 & 33,024 & $72.2 \pm 11.9$ & $20,058(60.7)$ & $5.8 \pm 2.0$ & 34.7 \\
\hline CKD (1-4) & 52,958 & $72.0 \pm 11.2$ & $34,892(65.9)$ & $5.5 \pm 2.1$ & 100 & 30,285 & $72.8 \pm 11.0$ & $19,769(65.3)$ & $5.7 \pm 2.0$ & 100 \\
\hline CTO & 52,884 & $65.6 \pm 12.0$ & $37,477(70.9)$ & $3.5 \pm 2.1$ & 15.9 & 25,361 & $68.2 \pm 12.0$ & $16,545(65.0)$ & $4.1 \pm 2.1$ & 26.2 \\
\hline Diabetes + CKD (3-4) + CTO & 2,764 & $71.1 \pm 10.6$ & $1,828(66.1)$ & $6.5 \pm 1.9$ & 100 & 3,060 & $70.8 \pm 10.4$ & 2,065 & $6.2 \pm 1.9$ & 100 \\
\hline
\end{tabular}

CKD, chronic kidney disease; IOCM, iso-osmolar contrast media; LOCM, low-osmolar contrast media; SD, standard deviation; CTO, chronic total occlusion; ECI, Elixhauser Comorbidity Index. * \% CKD is inclusive of CKD 1-4, but not CKD unspecified.

Table 2. Regression results for comorbidity groups

\begin{tabular}{lllllllll}
\hline & $\begin{array}{l}\text { IOCM, } \\
N\end{array}$ & \multicolumn{1}{l}{ LOCM, } \\
& $N$ & $\begin{array}{l}\text { LOCM } \\
\text { MARCE } \\
\text { rate, } \%\end{array}$ & ARR, $\%$ & $\begin{array}{l}\text { p value } \\
\text { associated } \\
\text { with ARR }\end{array}$ & RRR, \% & NNT \\
\hline Diabetes & 54,019 & 148,170 & 7.45 & 0.54 & 0.0024 & 7.2 & 186 \\
Heart failure & 33,024 & 83,740 & 13.06 & 0.69 & 0.0264 & 5.3 & 145 \\
CKD (1-4) & 30,285 & 52,958 & 15.89 & 1.83 & $<0.0001$ & 11.5 & 55 \\
CKD (3-4) & 18,471 & 30,168 & 17.15 & 2.36 & $<0.0001$ & 13.8 & 43 \\
Diabetes + CKD (3-4) & 18,306 & 11,645 & 18.44 & 3.52 & $<0.0001$ & 19.1 & 29 \\
\hline
\end{tabular}

ARR, absolute risk reduction; IOCM, iso-osmolar contrast media; LOCM, low-osmolar contrast media; RRR, relative risk reduction; NNT, number needed to treat; CKD, chronic kidney disease.

The incidence of MARCE in patients administered LOCM increased from $7.45 \%$ in patients with diabetes to $13.06 \%$ in patients with heart failure and $17.15 \%$ in patients with CKD 3-4, further rising to $18.44 \%$ in patients with a combination of diabetes and CKD 3-4 (Table 2). After multivariable modeling, use of IOCM was found to be associated with statistically significant lower incidence of MARCE than LOCM in patients with CKD 1-4, CKD $3-4$, diabetes or heart failure (Table 2; Fig. 2). ARR (Fig. 2a) associated with IOCM was $0.5 \%(p=0.0024)$ in the patient group with diabetes only and was associated with RRR (Fig. 2b) of 7.2\% (NNT = 186). These findings were also favorable for IOCM in patients with heart failure and CKD. The greatest ARR of $2.4 \%(p<0.0001)$ associated with a single risk factor was seen in CKD 3-4 patients $(\mathrm{RRR}=13.8 \%, \mathrm{NNT}=43)$. ARR associated with IOCM increased up to $3.5 \%(p<0.0001)$ in patients with combined risk of diabetes and CKD 3-4 $(\mathrm{RRR}=19.1 \%$, NNT $=29$ ).
Statistically significant risk reduction associated with use of IOCM was also found among patients who underwent revascularization for CTO (ARR $=1.6 \%[p<0.0001]$, RRR $=22.3 \%, \mathrm{NNT}=62)$. The patient cohort with diabetes, $\mathrm{CKD}$ $3-4$, and CTO procedure were found to have a borderline significant ARR of $2.77 \%$ ( $p=0.06)$. (Table 3; Fig. 2a).

\section{Discussion}

Iodinated contrast media are widely utilized in intraarterial and interventional procedures. With several agents available and limited real-world analyses evidence on outcome differences following the use of iodinated contrast available, choice of contrast is a decision that is largely left to individual facilities and practitioners.

This analysis was based on current clinical outcomes data (2012-2018) from a large claims database and included a variety of diagnostic and treatment procedures 
Fig. 2. $A R R$ (a) and $R R R$ (b) of $M A R C E$ incidence associated with use of IOCM compared to use of LOCM. IOCM, iso-osmolar contrast media; LOCM, low-osmolar contrast media; ARR, absolute risk reduction; RRR, relative risk reduction. a

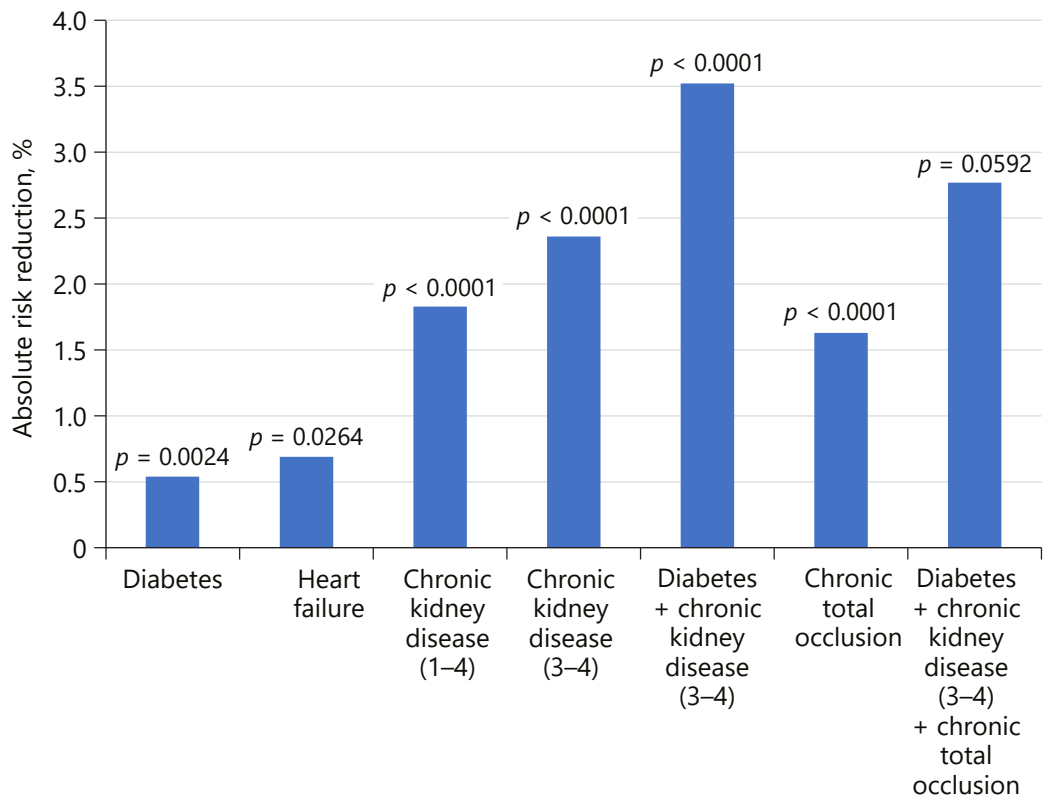

b

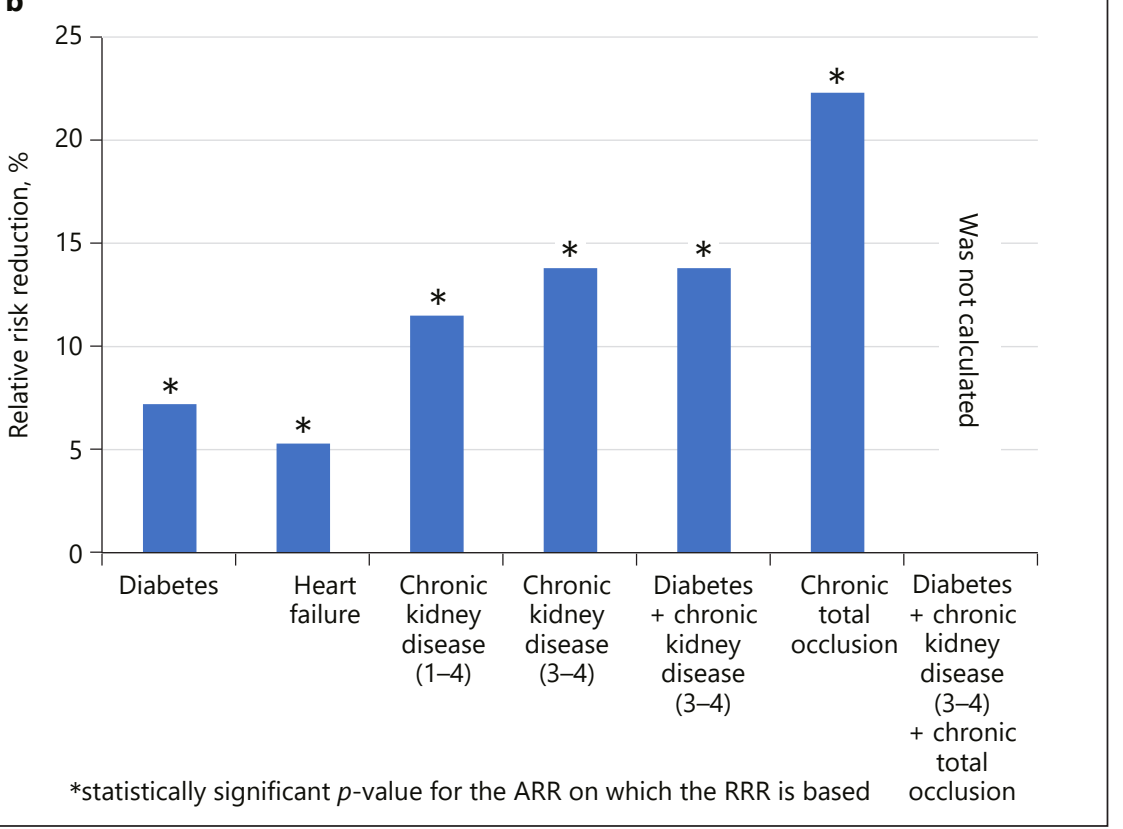

with intra-arterial contrast media administration. Within the inpatient visits there were 133,192 IOCM administrations and 402,821 LOCM. The incidence of MARCE following intra-arterial administration was investigated in several comorbidity-based at-risk subgroups. In comparison to LOCM, IOCM use resulted in a significantly lower risk of MARCE in subgroups of patients with diabetes, heart failure, $\mathrm{CKD}$, patients undergoing CTO procedures, and the combined group including those with
CKD stages 3-4 having diabetes. These outcomes further support prior studies evaluating IOCM and LOCM in patients undergoing angiography or other procedures associated with intra-arterial administration of contrast media [8-13]. Given the large volume of procedures done at most medical centers, the NNT values are clinically and institutionally meaningful.

Our data are cohesive with the findings of others in that CI-AKI has reliable determinants and thus is ame- 
Table 3. Regression results for CTO procedure and combination comorbidity

\begin{tabular}{|c|c|c|c|c|c|c|c|}
\hline & $\begin{array}{l}\text { IOCM, } \\
N\end{array}$ & $\begin{array}{l}\text { LOCM, } \\
N\end{array}$ & $\begin{array}{l}\text { LOCM } \\
\text { MARCE } \\
\text { rate, } \%\end{array}$ & ARR, \% & $\begin{array}{l}p \text { value } \\
\text { associated } \\
\text { with, ARR }\end{array}$ & RRR, \% & NNT \\
\hline CTO & 25,361 & 52,884 & 7.30 & 1.63 & $<0.0001$ & 22.3 & 62 \\
\hline Diabetes + CKD (3-4) + CTO & 3,060 & 2,764 & 19.08 & 2.77 & 0.0592 & - & - \\
\hline
\end{tabular}

ARR, absolute risk reduction; IOCM, iso-osmolar contrast media; LOCM, low-osmolar contrast media; RRR, relative risk reduction; NNT, number needed to treat; $\mathrm{CKD}$, chronic kidney disease; CTO, chronic total occlusion; RRR and NNT were not calculated due to borderline significant value of ARR.

nable to risk stratification and mitigation [2]. While there are many approaches to reducing the risk of CI-AKI, the choice of contrast agent which can be institutional, administrative, or protocol driven is one opportunity to reduce CI-AKI and thus have downstream benefits with respect to real-world outcomes [16].

Revascularization in patients with CTO is a challenging procedure, frequently necessitating longer procedural time and larger volume of iodinated contrast [17]. The CTO procedure in some circumstances where it is part of multivessel revascularization is an alternative to coronary artery bypass surgery which has been shown to add independent additional risks to the antecedent angiographic procedure [18]. Patients undergoing percutaneous coronary intervention for CTO with pre-existing CKD may experience adverse outcomes such as AKI $[19,20]$ and could benefit from a risk mitigation strategy comprising screening patients for risk factors, optimizing periprocedural hydration and managing the volume of contrast. Additionally, our investigation indicates that MARCE following a CTO procedure is associated with type of contrast media, suggesting the use of IOCM in these patients as part of risk mitigation strategy. Although the evaluation of the subset of at-risk patients with CKD and diabetes undergoing CTO revealed only borderline significant difference in MARCE rates between IOCM and LOCM, we speculate that this was likely due to combination of model complexity and the small sample size of this group. For the same reason, other comorbidity combinations such as CKD, diabetes, and heart failure were also not studied. Additional research in these patient subgroups is warranted.

Limitations of this study include those inherent to retrospective database studies, including the use of data for outcomes research which was collected for a separate purpose. In this study, administrative claims data were used; thus, the analysis relied on the ICD-9/10 coding of outcomes. This also meant that laboratory values were not available for the diag- nosis of AKI by serum creatinine levels, assessment of heart failure by ejection fraction, or other clinical conditions. While coding has been found to have a high degree of accuracy for comparative analysis [21], the use of ICD codes as an indicator of AKI may underestimate the occurrence of this event [22]. An additional limitation of this dataset is that patients are not tracked longitudinally; this limitation may have resulted in incorrect estimation of the true occurrence of MARCE. Finally, as exact contrast media volumes cannot be extracted from the Premier Healthcare Database, it was not possible to correlate higher MARCE rates to larger contrast volumes that have previously been associated with AKI [6] and have also been used in complex CTO procedures [17].

\section{Conclusion}

The use of IOCM in at-risk patients following intraarterial iodinated contrast media administration is associated with lower rates of MARCE than the use of LOCM. This difference is especially apparent in patients with a combination of CKD 3-4 and diabetes, and in patients undergoing revascularization procedures for CTO. Our NNT results suggest that institutional volumes of intra-arterial procedures in these at-risk patients may benefit from IOCM use in the pursuit of improved outcomes and enhanced safety. In at-risk patients, IOCM use may be considered as part of a multipronged strategy to mitigate adverse renal and cardiovascular outcomes.

\section{Statement of Ethics}

All data used to perform this analysis were de-identified and accessed in compliance with the Health Insurance Portability and Accountability Act. As a retrospective analysis of a de-identified database, the research was exempt from IRB review under 45 CFR 46.101(b) (4). 


\section{Conflict of Interest Statement}

McCullough P. is a consultant to GE Healthcare. Ng C.S. is a consultant to GE Healthcare. Ryan M. is an employee of MPR Consulting, which is a consultant to GE Healthcare, the study sponsor. Baker E.R. is an employee of CTI Clinical Trial and Consulting Services, which is a consultant to GE Healthcare, the study sponsor. Mehta R. has had financial relationships with Baxter, AM Pharma, Sanofi, Akebia, Intercept, Mallinckrodt, Biomerieux, Sphingotec, GE Healthcare, CHF solutions, Fresenius-Kabi, and Fresenius.

\section{Funding Sources}

GE Healthcare provided financial support for the study. The authors had full control of the design of the study, methods used, outcome parameters and results, analysis of data, and production of the written report.

\section{Author Contributions}

Each author contributed significantly to the submitted work. All authors contributed to the concept and design of the analysis. Ryan M. conducted the analysis. Baker E.R. drafted the manuscript; McCullough P., Ng C.S., Mehta R., and Ryan M. revised it for important intellectual content. All authors have read and approved the final version of the manuscript and agree to be accountable for all aspects of the work.

\section{Data Availability Statement}

The data that support the findings of this study are available from Premier Healthcare Database, but restrictions apply to the availability of these data and were used under license for the current study; therefore, they are not publicly available.

\section{References}

1 Kidney Disease: Improving Global Outcomes (KDIGO) Acute Kidney Injury Work Group. KDIGO clinical practice guideline for acute kidney injury. Kidney Inter Suppl. 2012;2:1138.

2 McCullough PA, Choi JP, Feghali GA, Schussler JM, Stoler RM, Vallabahn RC, et al. Contrast-induced acute kidney injury. J Am Coll Cardiol. 2016 Sep 27;68(13):1465-73.

3 Tsai TT, Patel UD, Chang TI, Kennedy KF, Masoudi FA, Matheny ME, et al. Contemporary incidence, predictors, and outcomes of acute kidney injury in patients undergoing percutaneous coronary interventions: insights from the NCDR Cath-PCI registry. JACC Cardiovasc Interv. 2014 Jan;7(1):1-9.

4 Ronco F, Tarantini G, McCullough PA. Contrast induced acute kidney injury in interventional cardiology: an update and key guidance for clinicians. Rev Cardiovasc Med. 2020 Mar 30;21(1):9-23.

5 Mehran R, Aymong ED, Nikolsky E, Lasic Z, Iakovou I, Fahy M, et al. A simple risk score for prediction of contrast-induced nephropathy after percutaneous coronary intervention: development and initial validation. J Am Coll Cardiol. 2004 Oct 6;44(7):1393-9.

6 Amin AP, Bach RG, Caruso ML, Kennedy KF, Spertus JA. Association of variation in contrast volume with acute kidney injury in patients undergoing percutaneous coronary intervention. JAMA Cardiol. 2017 Sep 1;2(9): 1007-12.

7 Azzalini L, Poletti E, Lombardo F, Laricchia A, Beneduce A, Moscardelli S, et al. Risk of contrast-induced nephropathy in patients undergoing complex percutaneous coronary intervention. Int J Cardiol. 2019 Sep 1;290: 59-63.

8 Aspelin P, Aubry P, Fransson SG, Strasser R, Willenbrock R, Berg KJ, et al. Nephrotoxic effects in high-risk patients undergoing angiog- raphy. N Engl J Med. 2003 Feb 6;348(6):4919.

9 Nie B, Cheng WJ, Li YF, Cao Z, Yang Q, Zhao YX, et al. A prospective, double-blind, randomized, controlled trial on the efficacy and cardiorenal safety of iodixanol versus iopromide in patients with chronic kidney disease undergoing coronary angiography with or without percutaneous coronary intervention. Catheter Cardiovasc Interv. 2008 Dec 1;72(7):958-65.

10 McCullough PA, Brown JR. Effects of intraarterial and intravenous iso-osmolar contrast medium (Iodixanol) on the risk of contrastinduced acute kidney injury: a meta-analysis. Cardiorenal Med. 2011;1(4):220-34.

11 Dong M, Jiao Z, Liu T, Guo F, Li G. Effect of administration route on the renal safety of contrast agents: a meta-analysis of randomized controlled trials. J Nephrol. 2012 May; 25(3):290-301.

12 Zhao F, Lei R, Yang S-K, Luo M, Cheng W, Xiao Y-Q, et al. Comparative effect of iso-osmolar versus low-osmolar contrast media on the incidence of contrast-induced acute kidney injury in diabetic patients: a systematic review and meta-analysis. Cancer Imaging. 2019 Jun 18;19(1):38.

13 McCullough PA, David G, Todoran TM, Brilakis ES, Ryan MP, Gunnarsson C. Iso-osmolar contrast media and adverse renal and cardiac events after percutaneous cardiovascular intervention. J Comp Eff Res. 2018 Apr; 7(4):331-41.

14 Premier Healthcare Database. White paper: date that informs and preforms. Charlotte, NC; 2020. Available from: https://products. premierinc.com/downloads/PremierHealthcareDatabaseWhitepaper.pdf.

15 Elixhauser A, Steiner C, Harris DR, Coffey RM. Comorbidity measures for use with administrative data. Med Care. 1998 Jan;36(1): $8-27$.
16 Keuffel E, McCullough PA, Todoran TM, Brilakis ES, Palli SR, Ryan MP, et al. The effect of major adverse renal cardiovascular event (MARCE) incidence, procedure volume, and unit cost on the hospital savings resulting from contrast media use in inpatient angioplasty. J Med Econ. 2018 Apr;21(4):356-64.

17 Demir OM, Lombardo F, Poletti E, Laricchia A, Beneduce A, Maccagni D, et al. Contrastinduced nephropathy after percutaneous coronary intervention for chronic total occlusion versus non-occlusive coronary artery disease. Am J Cardiol. 2018 Dec 1;122(11):1837-42.

18 Tecson KM, Brown D, Choi JW, Feghali G, Gonzalez-Stawinski GV, Hamman BL, et al. Major adverse renal and cardiac events after coronary angiography and cardiac surgery. Ann Thorac Surg. 2018 Jun;105(6):1724-30.

19 Azzalini L, Ojeda S, Demir OM, Dens J, Tanabe M, La Manna A, et al. Recanalization of chronic total occlusions in patients with versus without chronic kidney disease: the impact of contrast-induced acute kidney injury. Can J Cardiol. 2018 Oct;34(10):1275-82.

20 Lee WC, Wu PJ, Fang CY, Chen HC, Wu CJ, Fang HY. Impact of chronic kidney disease on chronic total occlusion revascularization outcomes: a meta-analysis. J Clin Med. 2021 Jan 23;10(3):440.

21 Amin AP, Spertus JA, Curtis JP, Desai N Masoudi FA, Bach RG, et al. The evolving landscape of impella use in the United States among patients undergoing percutaneous coronary intervention with mechanical circulatory support. Circulation. 2020 Jan 28; 141(4):273-84

22 Waikar SS, Wald R, Chertow GM, Curhan GC, Winkelmayer WC, Liangos O, et al. Validity of international classification of diseases, ninth revision, clinical modification codes for acute renal failure. J Am Soc Nephrol. 2006 Jun;17(6):1688-94. 